\title{
O PAPEL DA CICLOOXIGENASE-2 E DA PRESSÃO ARTERIAL NA LESÃO RENAL DE RATOS SUBMETIDOS À INFUSÃO CONTÍNUA DE ANGIOTENSINA II
}

\author{
Flávia Santin, ${ }^{1}$ Maria do Carmo Fernandez Vailati, ${ }^{2}$ Marcela Marcondes Pinto Rodrigues, ${ }^{3}$ \\ David Michelucci, ${ }^{4}$ Luis Cuadrado Martin ${ }^{5}$ e Denise Saretta Schwartz ${ }^{6}$ \\ 1. Professora adjunta I da Universidade Federal do Recôncavo da Bahia, Brasil. \\ E-mail:fla vet@ig.com.br \\ 2. Professor substituto da Universidade Estadual Paulista Júlio de Mesquita Filho \\ 3. Doutoranda da Universidade Estadual Paulista Júlio de Mesquita Filho \\ 4. Professor da UDESC, Departamento de Solos-Estatística \\ 5. Médico da Fundação para Desenvolvimento Médico e Hospitalar da FM Unesp-Botucatu \\ 6. Professor doutor da Universidade de São Paulo.
}

\section{RESUMO}

A angiotensina II (ANGII) é um vasoconstritor, entretanto, sua atuação na inflamação tem sido estudada. Objetivou-se verificar o estímulo da ciclooxigenase- 2 e da pressão arterial na lesão renal por ANGII. Para tanto, utilizaram-se ratos Wistar, distribuídos no grupo 1: controle, grupo 2: ANGII, grupo 3: ANGII + celecoxibe, grupo 4: celecoxibe. AANGII foi administrada via minibomba no subcutâneo dos animais durante 72 horas, sendo então realizada a eutanásia. A pressão arterial foi aferida antes da administração dos fármacos e antes da eutanásia. Colheram-se os rins dos animais para histopatologia e para imunoistoquímica, avaliando-se a expressão de COX-2. Concluiu-se que a ANGII promoveu lesões renais, independentemente dos valores da pressão arterial; ANGII associada ao celecoxibe levou ao aumento da pressão arterial. O celecoxibe não protegeu os rins contra as ações da angiotensina II.

PALAVRAS-CHAVES: Celecoxibe, nefrotoxicidade, pressão arterial. TO CONTINUOUS INFUSION OF ANGIOTENSIN II

Angiotensin II (ANGII) is a vasoconstrictor which action on inflammation has been studied. The objective of this study was to verify the stimulation of cyclooxigenase- 2 and blood pressure on renal damage by ANGII. Wistar rats were distributed into four groups, as follows: group 1 - control; group 2 - ANGII; group 3 - ANGII + celecoxib; and group 4 - celecoxib. The ANGII was infused in the animals by subcutaneous osmotic mini-pumps during 72 hours. After that, euthanasia was performed. The blood pressure was measured before the drugs administration and before the euthanasia. Histopathological and immunohistochemical evaluations were performed in kidneys to assess the expression of COX-2. It was concluded that ANGII caused renal damage, regardless of blood pressure values. ANGII associated with celeboxib increased blood pressure. Celecoxib did not protect the kidneys against ANGII activities.

KEYWORDS: Blood pressure, celecoxib, nephrotoxicity. 


\section{INTRODUÇÃO}

A angiotensina II (ANGII) é o principal polipeptídeo do sistema renina angiotensina aldosterona (SRAA) e exerce múltiplos efeitos fisiológicos, pela ativação de receptores específicos presentes nos vasos, no coração, nos rins, na adrenal, no cérebro, bem como no fígado (JACKSON \& GARRISON, 1996). A ANGII promove aumento da pressão arterial sistêmica de forma direta e indireta, uma vez que promove vasoconstrição periférica e aumento no volume circulante. Este aumento no volume é atribuído ao efeito direto da ANGII sobre os túbulos e sobre a estimulação da secreção de aldosterona, pelo córtex da adrenal. Esses dois efeitos levam à reabsorção de sódio, com subsequente reabsorção de água, que tendem a elevar o volume sanguíneo, um fator importante na regulação da pressão arterial em longo prazo (IRIGOYEN et al., 2001; MUSCARÁ et al., 2001). Por serem responsáveis pela produção da renina, com a subsequente formação da ANGII, os rins têm papel importante no controle da pressão arterial (PA) (ANDRADE et al., 2002).

Nos rins, o balanço de sal e água e a liberação de renina sofrem influência de substâncias denominadas prostaglandinas, que atuam como importantes mediadores do tônus vascular. $\mathrm{O}$ ácido aracdônico é o mais abundante e importante precursor das prostaglandinas, produzidas por duas vias enzimáticas: a ciclooxigenase e a lipoxigenase (CARVALHO et al., 2004). A enzima ciclooxigenase (COX), que possui duas isoformas - a COX- 1 e a COX-2 -, inicia a conversão do ácido aracdônico em prostaglandinas e tromboxano (CHENG \& HARRIS, 2004). A isoforma COX-2 é principalmente induzida em processos inflamatórios e de carcinogênese, entretanto tem sua expressão detectada também em tecido normal de rim (HARRIS, 2002; MUNGAN, et al., 2006). Os produtos da COX podem ter importante papel na patogênese de nefropatias, como, por exemplo, em doenças como lúpus eritematoso sistêmico e glomeruloesclerose, ocorre um aumento na expressão das isoformas COX-1 e COX-2 (GONÇALVES et al., 2004; JAIMES et al., 2005).

Os anti-inflamatórios não esteroidais (AINES) encontram-se entre os fármacos mais utilizados em todo o mundo, sendo desenvolvidos para tratar condições dolorosas e inflamatórias, agudas e crônicas (CRUZ et al., 2000). A atividade anti-inflamatória dos AINES é mediada principalmente pela inibição da biossíntese das prostaglandinas. Os inibidores seletivos da COX-2, ou os denominados coxibes, foram desenvolvidos para inibir a síntese das prostaciclinas produzidas pela isoenzima COX-2, induzida em locais de inflamação. Esses medicamentos ligam-se seletivamente ao sítio ativo da enzima COX-2 para bloquear sua ação.

Eles exercem efeitos analgésicos, antipiréticos e anti-inflamatórios semelhantes aos AINES não específicos, porém com menor efeito colateral gastrointestinal, sendo o celecoxibe um exemplo dessa classe de fármacos (FITZGERALD \& PATRONO, 2001; WAGNER et al., 2006). Uma vez que há a expressão da COX-2 de forma constitutiva nos rins normais, atenção é voltada para possíveis ações deletérias dos coxibes nestes órgãos (BREYER \& HARRIS, 2001; BRATER, 2002). BREYER et al. (2001) e FOSSLIEN (2005) chamam a atenção para a capacidade de os coxibes promoverem diminuição da excreção renal de sódio, com respectivo aumento na retenção de água, edema celular e ganho de peso, além da diminuição de prostaciclinas, fatores que promovem hipertensão arterial.

Objetiva-se com o protocolo experimental de infusão contínua de ANGII durante 72 horas avaliar se a lesão renal induzida pela ANGII tem como mecanismo de ação o estímulo da ciclooxigenase-2 e avaliar a relação entre pressão arterial, intensidade e tipo de lesão renal.

\section{MATERIAL E MÉTODOS}

Utilizaram-se 39 ratos Wistar, machos, adultos, com aproximadamente $250 \mathrm{~g}$ de peso corpóreo, provenientes do CEMIB- Unicamp, Campinas, SP. Mantiveram-se os animais no biotério de experimentação do Departamento de Clínica Médica da Faculdade de Medicina de Botucatu, em ambiente com controle de luz (ciclos de doze horas) e temperatura constante $\left(25^{\circ} \mathrm{C}\right)$, recebendo dieta padrão e água ad libitum. Os procedimentos realizados nos animais foram executados no Centro de Pesquisa da Faculdade de Medicina, Laboratório Experimental do Departamento de Clínica Médica, UNESP-Botucatu, e foram aprovados pela Câmara de Ética em Experimentação Animal como protocolo de número 109/07. Dividiram-se aleatoriamente os animais em quatro grupos: grupo 1: ratos 
controle $(\mathrm{n}=10)$, grupo 2: ratos tratados com ANGII (n =11); grupo 3: ratos tratados com iCOX-2 (Celecoxibe, Celebra $\left.^{\circledR}\right) 10 \mathrm{mg} / \mathrm{kg} / \mathrm{dia}$, via gavagem); $\mathrm{n}=9$ e grupo 4: ratos tratados com ANGII + iCOX-2 (Celecoxibe, Celebra $\left.^{\circledR}\right) 10 \mathrm{mg} / \mathrm{kg} / \mathrm{dia}$, via gavagem; $\mathrm{n}=9$.

Induziu-se a lesão renal por meio de infusão contínua de angiotensina $\mathrm{II}^{1}(150 \eta \mathrm{g} / \mathrm{min} / \mathrm{SC})$. Essa dosagem foi descrita por causar lesão renal de aspecto inflamatório (VAILATI et al., 2010). A infusão contínua de ANGII foi administrada por meio de minibombas osmóticas (modelo \#2002, ALZA Corp) implantadas no subcutâneo do animal (JAIMES et al., 2005; RUPEREZ et al., 2005). Sedaram-se os ratos com a associação de quetamina ${ }^{2}$ (50 mg/ Kg IM) e xilazina ${ }^{3}$ (10 mg/ $\mathrm{Kg}$ IM), sendo a minibomba implantada por meio de pequena incisão no dorso dos animais. Esse dispositivo libera $0,5 \mu \mathrm{L} /$ hora de solução. Assim, as minibombas foram preenchidas com solução contendo ANGII, para oferecer ao animal a dose de $150 \mathrm{\eta g} / \mathrm{min}$.

Utilizou-se, como inibidor seletivo da cicloxigenase-2 (iCox2), o celecoxibe, ${ }^{4}$ na dose de $10 \mathrm{mg} / \mathrm{kg} /$ dia, via gavagem (PINHEIRO \& CALIXTO, 2002). A administração do iCox 2 foi iniciada três dias antes do início da infusão contínua de ANGII, e continuou até o final do experimento, com o objetivo de ser observada a influência da inibição da COX-2 no aparecimento de lesões induzidas pela ANGII.

A pressão arterial sistólica caudal foi obtida aproximadamente 24 horas antes do início dos tratamentos, com o intuito de ser obtido o valor da pressão arterial basal dos animais, e no dia da eutanásia, para ser observada a influência de cada tratamento na pressão arterial dos animais, utilizando-se o sistema de pressão caudal com polígrafo modelo RS3200-Gould e o esfigmomanômetro Narco Bio-Systems PE-300.

As alterações histológicas dos glomérulos, túbulos, interstício e vasos foram graduadas semiquantitativamente segundo escala de 0 a $3+$ : ausência de lesão $=0$, lesão discreta $=1+$, lesão moderada $=2+$, lesão intensa $=3+$. Em relação às lesões vasculares, consideraram-se lesão discreta $=$ um a cinco vasos comprometidos, lesão moderada = lesão em seis a dez vasos e lesão intensa $=$ mais de dez vasos com lesão.

1. Sigma ${ }^{\circledR}$ - Missouri-USA

2. Katamin ${ }^{\circledR}$ Lab Cristália

3. Rompum ${ }^{\circledR}$ - Bayer

4. Celebra ${ }^{\mathbb{Q}}$ - Pfizer
As alterações semiquantificadas foram avaliadas em glomérulos quanto à proliferação epitelial, mesangial e endotelial, matriz mesangial, necrose, esclerose e aderências do tufo glomerular com a cápsula de Bowman. Avaliaram-se os túbulos quanto à degeneração celular, necrose, regeneração e atrofia. No interstício, avaliou-se a presença de edema e infiltrado inflamatório e fibrose. Os vasos foram avaliados quanto à presença de fibroelastose das artérias, arteriolosclerose hialina, endarterite proliferativa, necrose fibrinoide e congestão. Definiram-se, como índice de lesão glomerular (ILG), túbulo-intersticial (ILT-I), e como vascular (ILV), a somatória dos sinais positivos $(+)$ atribuídos a cada uma das alterações mencionadas. A soma desses índices foi o índice de lesão renal total (ILR). Avaliou-se a expressão de COX-2 renal em cortes histológicos de rins, preparados por técnica de imunoistoquimica, utilizando-se o kit Monoclonal Mouse Anti-Human COX-2 (Cyclooxygenase-2) Clone: CX-294 (Dako ${ }^{\circledR}$ ).

Os índices de lesões glomerular (ILG), túbulointersticial (ILT-I), vascular (ILV), renal total (ILRT), e a marcação positiva para COX-2 foram analisadas pelo teste de Kruskal-Wallis, com teste de comparações múltiplas de Dunn (DeMUTH, 1999). Avaliou-se a variável pressão arterial final por meio de análise de co-variância com a pressão inicial utilizada como covariável (SNEDECOR \& COCHRAN, 1989). Também foi determinado o grau de correlação entre a variável pressão arterial final e índice de lesão renal no exame histopatológico, pela análise de correlação simples de Spearman (DeMUTH, 1999), para determinar a relação entre pressão arterial e grau de lesão renal. A probabilidade de significância mínima foi estabelecida em $5 \%(p<0,05)$.

\section{RESULTADOS E DISCUSSÃO}

A infusão subcutânea contínua de ANGII não causou hipertensão arterial neste protocolo experimental, sendo que apenas o grupo ANGII+Celecoxibe teve valores de PA estatisticamente significantes, contrariando os achados de HENEGAR et al. (1995) e VAILATI et al. (2010), que, com esta mesma dose, obtiveram elevação na pressão arterial sistólica caudal. Uma possível explicação para estes achados consiste no mecanismo de ação pressórica da ANGII, que é dose 
e tempo dependente (LEVER et al., 1992; EDGLEY et al., 2003). Sendo assim, a dose deste protocolo experimental poderia potencialmente promover aumento dos valores de PA, caso fosse administrada durante um período maior de dias. Os achados do presente estudo são corroborados por BREYER et al. (2001) e FOSSLIEN (2005), que descrevem sobre a capacidade de os anti-inflamatórios seletivos COX-2 promoverem hipertensão arterial. Esta seria induzida, provavelmente, pela retenção tubular de sódio que estes medicamentos promovem, com o consequente aumento de volume circulante (KUMMER \& COELHO, 2002), ou pela diminuição de prostaciclinas responsáveis pela vasodilatação arterial (HÖRCHEL et al., 2002). Podese então afirmar que a administração de celecoxibe a pacientes que apresentam níveis séricos aumentados de ANGII deve ser realizada com extrema cautela, ou até mesmo evitada.

Houve a indução de lesões renais como era esperado, uma vez que a dose de 150 ๆg/min já havia sido utilizada em outros trabalhos, que também obtiveram lesão renal (VAILATI et al., 2010). Os grupos que receberam ANGII apresentaram o maior índice de lesão renal total (ILT= de 9 a 11). A análise morfológica mostrou lesões vasculares associadas às lesões degenerativas e de necrose dos túbulos, sendo o conjunto de achados compatível com microangiopatia trombótica (Figura 1).

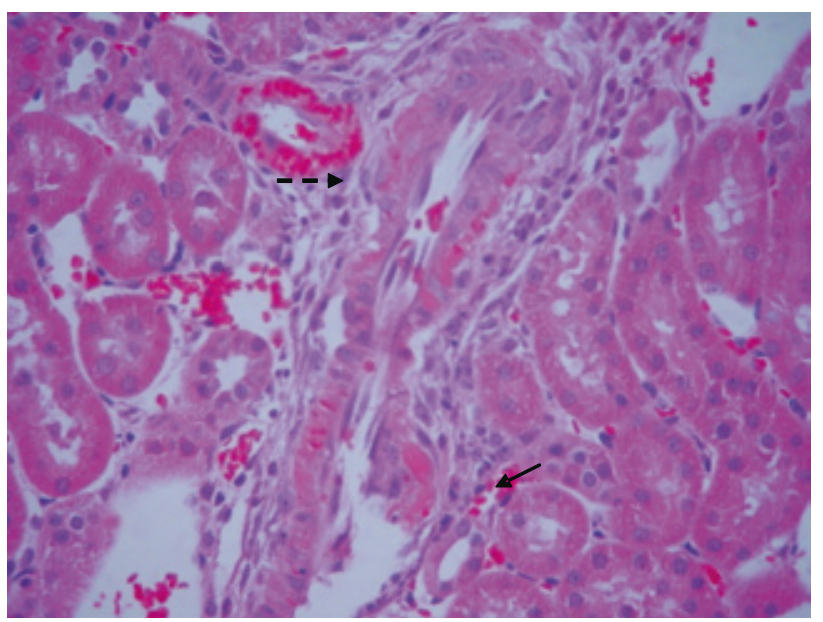

FIGURA 1. Rim de rato Wistar tratado com ANGII: presença de necrose fibrinoide segmentar $(\rightarrow)$ e presença de necrose com hemorragia (-- $)$ em artéria (HE-400x).
Essas lesões são comumente observadas em casos de hipertensão arterial maligna, como descrito por LINDER (2000) e CAMARGO \& EUGUI (2007). KOBORI et al. (2007) descrevem que o aumento da PA pode ter sido o responsável pela indução de lesões renais, caracterizadas por glomeruloesclerose e atrofia tubular. LONG et al. (2004) e NAKAMURA et al. (2005), concordantes com essa relação entre PA e lesão renal, administraram medicamentos anti-hipertensivos, inibidores da enzima de conversão de angiotensina (iECA), observando melhora progressiva das lesões renais, à medida que a $\mathrm{PA}$ foi normalizada.

Entretanto, os achados do presente trabalho demonstram a capacidade de a ANGII promover lesões renais, independentes de valores pressóricos, à semelhança de SOROOSHIAN (2000) e RUPÉREZ et al. (2003), que descreveram sobre a indução de alterações renais através de mecanismos independentes de pressão arterial.

BIDANI \& GRIFFIN (2004) e WOLF \& RITZ (2005) observaram em diversos trabalhos experimentais uma proteção renal mais consistente em grupos de animais que receberam iECA em relação a outros tipos de anti-hipertensivos, embora todos os medicamentos tenham obtido sucesso no controle da pressão arterial, corroborando a afirmação de que a lesão renal via ANGII é independente de ação pressórica.

Assim, da mesma maneira que o bloqueio do sistema renina é capaz de proteger os rins de forma independente da redução pressórica, no presente estudo ficou demonstrada uma lesão direta da ANGII, também independente de sua ação pressórica. Vale ressaltar que, exceto um animal, todos os outros não tiveram aumento de PA, concordando com os achados de lesão vascular, que demonstraram a capacidade de a ANGII induzir lesão renal independente de fator pressórico.

Em relação aos nove animais do grupo ANGII+Celecoxibe, quatro tiveram imunomarcação positiva para a COX-2, embora este aumento não tenha sido estatisticamente significante, em relação ao grupo controle (Figura 2). Este achado é um paradoxo, uma vez que se trata de grupo que recebeu um antiinflamatório inibidor seletivo de COX-2, e o esperado seria a não expressão dessa enzima. Uma possível explicação para este fenômeno está no fato de que, como houve a associação da ANGII, esta promoveu vasoconstrição local, que levou a hipóxia, com con- 
sequente dano celular. Estudos in vitro com AINES, em situações de hipóxia, demonstraram um aumento da COX-2 nos monócitos estudados, sendo que este evento foi atribuído à inibição da prostaglandina PGE2, que estimulou a produção de TNF- $\alpha$, que, por sua vez, estimulou a expressão da COX-2 (FOSSLIEN, 2005). Observou-se também que os quatro animais tiveram lesão detectada no exame histopatológico, demonstrando necrose vascular variando entre $1+$ até $3+$, em um até cinco vasos. Sendo assim, pode-se sugerir que houve uma provável lesão por hipóxia nestes animais, tendo sido induzida pela ANGII.

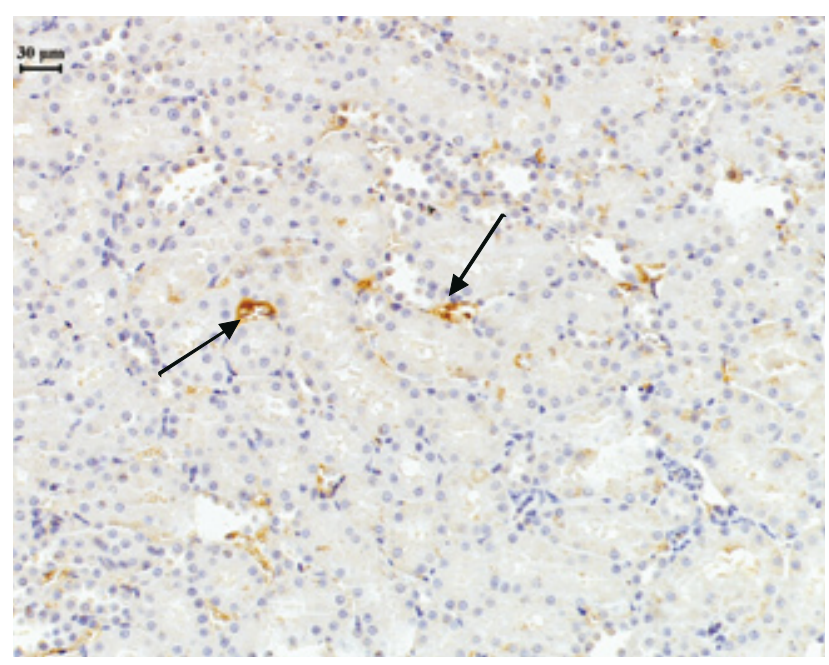

FIGURA 2. Marcação positiva $(\rightarrow)$ para COX-2 em rim de rato Wistar tratado com ANGII+ celecoxibe (200x).

Entre os dez animais do grupo celecoxibe, cinco deles tiveram expressão da enzima COX-2, de forma paradoxal ao esperado, mas também não estatisticamente significante. Uma possível explicação para este achado seria um comportamento bioquímico semelhante ao que ocorre no coração, quando há a administração de medicamentos $\beta$-bloqueadores, pois, quando administrados, estes medicamentos acabam promovendo uma up-regulation dos seus receptores (BATLOUNI \& RAMIRES, 1999). Dos 21 animais dos grupos controle e ANGII apenas cinco tiveram imunomarcação positiva para a COX-2, marcação esta que pode ser justificada pela presença da enzima COX-2 de forma constitutiva nos rins de ratos, como demonstrado por LIM et al. (2003) e HARRIS et al. (2008).
Considerando o protocolo experimental e as condições do estudo, os resultados permitiram concluir que a angiotensina II promoveu lesões renais, independentemente dos valores da pressão arterial e que a associação de ANGII e celecoxibe levou ao aumento da pressão arterial. O celecoxibe não protegeu os rins contra as ações da angiotensina II e, portanto, o mecanismo de ação da ANGII foi independente da expressão da COX-2.

\section{COMITÊ DE ÉTICA}

As técnicas utilizadas e o manejo dos animais foram aprovados pela Câmara de Ética em Experimentação Animal da FMVZ- UNESP- Botucatu, com o protocolo de número 109/07.

\section{AGRADECIMENTOS}

À Fundação de Amparo à Pesquisa do Estado de São Paulo (FAPESP - processo 2004/07661-3) e à Coordenação de Aperfeiçoamento Pessoal de Ensino Superior (CAPES), pelo apoio financeiro a esta pesquisa e pelas bolsas concedidas, respectivamente.

\section{REFERÊNCIAS}

ANDRADE, A. Q.; CASARINI, D. E.; SCHOR, N.; BOIM, M. A. Characterization of renin mRNA expression and enzyme activity in rat and mouse mesangial cells. Brazilian Journal of Medical and Biological Research, v. 35, n. 1, p. 17-24, 2002.

BATLOUNI, M.; RAMIRES, J. A. Bloqueadores beta-adrenérgicos. In: Farmacologia e terapêutica cardiovascular. São Paulo: Atheneu, 1999. p. 215-243.

BIDANI, A. K.; GRIFFIN, K. A. Pathophysiology of hypertensive renal damage implications for therapy. Hypertension, v. 44, p. 595-601, 2004.

BRATER, D. C.; HARRIS, C.; REDFERN, J. S.; GERTZ, B. J. Renal effects of COX-2-selective inhibitors. American Journal Nephrology, v. 21, n. 1, p. 1-15, 2002.

BREYER, M. D.; HAO, C.; ZHONGHUA, Q. COX2 inhibitors and kidney. Current Opinion in Critical Care, n. 7, p. 393-400, 2001.

BREYER, M. D.; HARRIS, R. C. Cycloxigenase 2 and the kidney. Current Opinion in Nephrology and Hypertension, v. 10, p. 89-98, 2001. 
CAMARGO, J. L. V.; ELGUI, O. D. Patologia geral: abordagem multidisciplinar. Rio de Janeiro: Guanabara Koogan, 2007. 204 p.

CARVALHO, W. A.; CARVALHO, R. D. S.; SANTOS, F. R. Analgésicos inibidores específicos da ciclooxigenase-2: avanços terapêuticos. Revista Brasileira de Anestesiologia, v. 54, n. 3, p. 448-464, 2004.

CHENG, H. F.; HARRIS, R. C. Cyclooxigenases, the kidney, and hypertension. Hypertension, v. 43, p. 525-530, 2004.

CRUZ, M. L.; LUNA, S. P. L.; SILVA, J.; IAMAGUTE, P.; CROCCI, A.; TAKAHIRA, R. K. Efeitos do flunixin, ketoprofeno, carprofeno, brupenorfina e placebo para analgesia pós-operatória em cães submetidos à osteossíntese de fêmur. A Hora Veterinária, v. 114 , p. $19-25,2000$.

DE MUTH, J. Basic statistics and pharmaceutical statistical applications. New York: Marcel Dekker Inc., 1999. 591 p.

EDGLEY, A. J.; MICHELLE, M. A.; ANDERSON, W. P. Evidence for renal vascular remodeling in angiotensin II-induced hypertension. Journal of Hypertension, v. 21, p. 1401-1406, 2003.

ELMARAKBY, A. A.; QUIGLEY, J. E.; IMIG, J. D.; POLLOCK, J. S.; POLLOCK, D. M. TNF- $\alpha$ inhibition reduces renal injury in DOCA-salt hypertensive rats. American Journal of Physiology: Regulatory, Integrative and Comparative Physiology, v. 294, p. R76-R83, 2007.

FITZGERALD, G. A.; PATRONO, C. The coxibs, selective inhibitors of cyclooxygenase-2. The New England Journal of Medicine, v. 345, n. 6, p. 433-442, 2001.

FOSSLIEN, E. Review: cardiovascular complications of nonsteroidal anti-inflammatory drugs. Annals of Clinical and Laboratory Science, v. 35, n. 4, p. 347-385, 2005.

GONÇALVES, A. R. R.; FUJIHARA, C. K.; MATTAR, A. L.; MALHEIROS, D. M. A. C.; NORONHA, I. L.; NUCCI, G.; ZATZ, R. Renal expression of COX-2, ANGII, and AT1 receptor in remnant kidney: strong renoprotection by therapy with losartan and a nonsteroid anti-inflammatory. American Journal of Physiology: Renal Physiology, v. 286, p. F945-F954, 2004.

GRAUER, G. F. Canine glomerulonephritis: new thoughts on proteinúria and treatment. Journal of Small Animal Practice, v. 46, p. 469-478, 2005.

HARRIS, R. C. Cyclooxygenase-2 inhibition and renal physiology. American Journal of Cardiology, v. 89, p. 10D-17D, 2002.

HARRIS, R. C. An update on cyclooxygenase-2 expression and metabolites in the kidney. Current Opinion in Nephology and Hypertension, v. 17, p. 64-69, 2008.
IRIGOYEN, M. C.; COLOMBO, F. M. C.; KRIEGER, E. M. Controle cardiovascular: regulação reflexa e papel do sistema nervoso simpático. Revista Brasileira de Hipertensão, n. 8, p. 55-62, 2001.

JACKSON, E. K.; GARRISON, J. C. Renina e angiotensina. In: GOODMAN, L.S.; GILMAN, A. As bases farmacológicas da terapêutica. 9. ed. Rio de Janeiro: Guanabara Koogan, 1996. p. 536-554.

JAIMES, E. A.; TIAN, R.; PEARSE, D.; RALI, L. Up-regulation of glomerular cox-2 by angiotensina ii: role of reactive oxygen species. Kidney International, v. 68, p. 2143-2153, 2005.

KUMMER, C. L.; COELHO, T. C. R. B. Anti-inflamatórios não esteroidais inibidores da ciclooxigenase-2 (COX-2): aspectos atuais. Revista Brasileira de Anestesiologia, v. 52, n. 4, p. 498$512,2002$.

LINDER, D. Kidney and urinary tract. In: Pathology: a color atlas. Philadhelphia: Mosby, 2000. 474 p.

LONG, D. A.; PRICE, K. L.; ACOSTA, J. H.; JOHSON, R. J. How does angiotensin II cause renal injury? Hypertension, v. 43, p. $722-723,2004$

MUNGAN, M. U.; GUREL, D.; CANDA, A. E.; TUNA, B.; YORUKOGLU,K.; KIRKALI, Z. Expresión of cox2 in normal and pyelonephritic kidney, renal renal intraepithelial neoplasia, and renal cell carcinoma. European Urology, n. 1140, p. 1-6, 2006.

MUSCARÁ, M. N.; RIBEIRO, W. Características farmacocinéticas de antagonistas de cálcio, inibidores da ECA e antagonistas de angiotensina II em humanos. Revista Brasileira de Hipertensão, n. 8 , p. $114-24,2001$.

NAKAMURA, A.; JOHNS, E. J.; IMAIZUMI, A.; YANAGAWA, Y.; KOSHAKA, T. Effect of adrenoceptor activation and angiotensin II on tumor necrosis factor and interleukine 6 gene transcription in the rat renal resident machrophage cells. Cytokine, v. 11, n. 10, p. 759-765, 2005.

PINHEIRO, R. M.; CALIXTO, J. B. Effect of the selective COX-2 inhibitors, celecoxib and rofecoxib in rat acute models of inflammation. Inflammation Research, v. 51, p. 603-610, 2002.

QI, Z.; CAI, H.; MORROW, J. D.; BREYER, M. D. Differentiation of cyclooxygenase-1 and 2- derived prostanoids in mouse kidney and aorta. Hypertension, v. 48, n. 2, p. 323-328, 2006.

RUPÉREZ, M.; LÓPEZ, E. S.; COLIO, L. M. B.; ESTEBAN, V.; VITA, J. R.; PLAZA J. J.; EGIDO, J.; ORTEGA, M. R. The rho-kinase pathway regulates AII-induced renal damage. Kidney International,v. 99, p. S39-45, 2005. 
RUPÉREZ, M.; ORTEGA, M. R.; ESTEBAN, V.; LORENZO, O.; MEZZANO, S.; PLAZA, J. J.; EGIDO, J. Angiotensin ii increases connective tissue growth factor in the kidney. American Journal of Pathology, v. 163, n. 5, p. 1937-1947, 2003.

SNEDECOR, G. W.; COCHRAN, W. G. Statistical Methods. Yowa: Iowa State University Press, 1989. p. 491.

SOROOSHIAN, M.; OLSON, J. L.; MEYER, T.W. Effect of angiotensin II blockade on renal injury in mineralocorticoid-salt hypertension. Hypertension, v. 36, p. 569-574, 2000.

VAILATI, M. C. F.; ROCHA, N. S.; MATSUBARA, L. S.; PADOVANI, C. R.; SCHWARTZ, D. S.; MATSUBARA, B. B. Protective effects of carvedilol on systemic vascular damage induced by angiotensin II: Organ-specific effects independent of antihypertensive effects. Medical Science Monitor, v. 16, n. 1, p. BR6-10, 2010.

WAGNER, W.; KHANNA, P.; FURST, D. E. Anti-inflamatórios não esteroidais, fármacos anti-reumáticos, modificadores de doença, analgésicos não opióides e fármacos utilizados na gota. In: KATZUNG, B. G. Farmacologia básica e clínica. Rio de Janeiro: Guanabara-Koogan, 2006. p. 481-504.

WOLF. G.; RITZ, E. Combination therapy with ACE inhibitors and angiotensin II receptor blockers to halt progression of chronic renal disease: pathophysiology and indications. Kidney International, v. 42, n. 3, p. 799-812, 2005.

Protocolado em: 26 ago. 2008. Aceito em: 27 jul. 2010. 